\title{
Synthesis and Photocatalytic Activity of Magnetically Recoverable Core-Shell Nanoparticles
}

\author{
Zhen Peng, ${ }^{1}$ Hua Tang, ${ }^{1}$ Yao Tang, ${ }^{2}$ Ke Fu Yao, ${ }^{2}$ and Hong Hong Shao ${ }^{1}$ \\ ${ }^{1}$ School of Materials Science and Engineering, Jiangsu University, Zhenjiang 212013, China \\ ${ }^{2}$ School of Materials Science and Engineering, Tsinghua University, Beijing 100084, China \\ Correspondence should be addressed to Zhen Peng; peng@ujs.edu.cn and Ke Fu Yao; kfyao@tsinghua.edu.cn
}

Received 7 March 2014; Accepted 25 March 2014; Published 14 April 2014

Academic Editor: Jiaguo Yu

Copyright (C) 2014 Zhen Peng et al. This is an open access article distributed under the Creative Commons Attribution License, which permits unrestricted use, distribution, and reproduction in any medium, provided the original work is properly cited.

\begin{abstract}
$\mathrm{TiO}_{2} / \mathrm{SiO}_{2} / \mathrm{Fe}_{3} \mathrm{O}_{4}$ (TSF) core-shell nanoparticles with good photocatalytic activity that are capable of fast magnetic separation have been successfully prepared by chemical coprecipitation and two-step sol-gel process. The as-prepared TSF nanoparticles were calcined at high temperature in order to transform the amorphous titanium dioxide into a photoactive crystalline phase. The calcined nanoparticles are composed of a $\mathrm{Fe}_{3} \mathrm{O}_{4}$ core with a strong response to external magnetic fields, a $\mathrm{SiO}_{2}$ intermediary layer, and $\mathrm{TiO}_{2}$ outshell. Vibration sample magnetometer (VSM) analysis confirms the superparamagnetism of calcined nanoparticles, which can enhance the recoverable properties of the novel photocatalyst. When the $\mathrm{TiO}_{2} / \mathrm{SiO}_{2} / \mathrm{Fe}_{3} \mathrm{O}_{4}$ core-shell nanoparticles are added to the crude oily wastewater, they exhibit high photocatalytic activity in the degradation of crude oily wastewater. The oil concentration could be reduced to lower than $30 \mathrm{ppm}$ within 20 minutes for the case of initial oil concentration less than $350 \mathrm{ppm}$. It has been found that the TSF nanoparticles could be easily separated from the wastewater and withdrawn by using an external magnetic field. The recovered TSF nanoparticles possess high efficiency in the degradation of crude oily wastewater even after three times successive reuse. The present results indicate that TSF core-shell nanoparticles possess great application perspectives in the degradation of crude oily wastewater.
\end{abstract}

\section{Introduction}

Photocatalytic technology offers a facile and cheap method for removing inorganic and organic pollutants from wastewater [1-4], since most pollutants could be degraded or mineralized by use of photocatalytic degradation technology [5-7]. Photocatalysts used in UV or near-UV light activated processes are semiconductor materials such as $\mathrm{TiO}_{2}, \mathrm{ZnO}$, and CdS [8,9]: among them, the nanosized titanium dioxide is one of the most widely used photocatalysts due to its high photocatalytic activity, low cost, good stability, and nontoxic nature [10-12].

When $\mathrm{TiO}_{2}$ photocatalyst is employed in the degradation of wastewater by photocatalytic process, it could be suspended in wastewater directly or be supported on substrate materials firstly and then immersed in wastewater with substrates. Nanosized $\mathrm{TiO}_{2}$ photocatalyst can either be suspended in wastewater or be supported on substrate materials. Nanosized $\mathrm{TiO}_{2}$ photocatalyst immobilized on substrate materials (such as glass, zeolite, silica, and ceramic) would benefit its separation from the wastewater. However, the activity of $\mathrm{TiO}_{2}$ photocatalyst in the fixed state is reduced to a considerable extent because the effective surface area of $\mathrm{TiO}_{2}$ photocatalyst decreases dramatically after the immobilization of $\mathrm{TiO}_{2}$. In addition, $\mathrm{TiO}_{2}$ photocatalyst may easily fall off the substrate materials, which make their complete recovery from wastewater difficult $[13,14]$. So developing recoverable $\mathrm{TiO}_{2}$ photocatalysts with high photocatalytic activity is meaningful and imperative. It has been reported that magnetic separation provides a suitable solution of this problem for removing $\mathrm{TiO}_{2}$ photocatalysts from wastewater and reusing by applying external magnetic field [15-21]. The combination of $\mathrm{TiO}_{2}$ photocatalyst and magnetic oxide nanoparticles $\left(\mathrm{Fe}_{3} \mathrm{O}_{4}, \gamma-\mathrm{Fe}_{2} \mathrm{O}_{3}\right.$, and $\left.\alpha-\mathrm{Fe}_{2} \mathrm{O}_{3}\right)$ 
may enhance the separation and recoverable property of nanosized $\mathrm{TiO}_{2}$ photocatalyst. However, compared with nanosized $\mathrm{TiO}_{2}$, the magnetic oxide nanoparticles are much more unstable, especially under acidic conditions. Beydoun et al. $[22,23]$ reported a photo-dissolution phenomenon, which was found in the coating anatase $\mathrm{TiO}_{2}$ directly onto magnetite. Electronic interactions will occur between $\mathrm{TiO}_{2}$ and magnetite core, which not only deteriorates the photocatalytic activity of $\mathrm{TiO}_{2}$, but also changes the magnetic properties of magnetite core. Furthermore, the preparing process of $\mathrm{TiO}_{2}$ photocatalyst usually involves a high temperature annealing, magnetic oxide nanoparticles such as $\mathrm{Fe}_{3} \mathrm{O}_{4}$ or $\gamma-\mathrm{Fe}_{2} \mathrm{O}_{3}$, if treated concurrently, may transform to antiferromagnetic $\alpha-\mathrm{Fe}_{2} \mathrm{O}_{3}$, which will reduce the property of magnetic response [24]. A suitable solution of this problem is the utilization of a passive interlayer $\mathrm{SiO}_{2}$ between the magnetic core and $\mathrm{TiO}_{2}$ shell. It has been found the $\mathrm{SiO}_{2}$ layer promotes the photocatalytic activity of the catalyst by decreasing the negative effect of magnetic core $[25,26]$. However, in prior studies the photocatalytic activity of combined photocatalysts did not show much improvement when compared with anatase-form nanoparticles, probably because the size of $\mathrm{TiO}_{2}$ shell and magnetic core was not controlled reasonably.

In present study, the TSF core-shell magnetic nanoparticles, which constitute a $\mathrm{Fe}_{3} \mathrm{O}_{4}$ layer, a $\mathrm{SiO}_{2}$ intermediary layer, and a $\mathrm{TiO}_{2}$ outer shell, have been successfully prepared. The TSF core-shell magnetic photocatalysts exhibit high photocatalytic efficiency, which is the same as the wellknown commercial photocatalyst P25, a mixture of $80 \%$ anatase and $20 \%$ rutile form of $\mathrm{TiO}_{2}$ produced by Degussa Chemical Company (Germany). As a result, the TSF coreshell magnetic photocatalysts can be efficiently recovered from the solution by using external magnetic field for many times without significant loss of photocatalysts and photocatalytic activity. The photocatalytic activity of the as-prepared TSF core-shell magnetic nanoparticles and the recovered ones has been studied by photocatalytic experiments in the degradation of crude oily wastewater.

\section{Experiment}

2.1. Synthesis of $\mathrm{Fe}_{3} \mathrm{O}_{4}$ Core. The $\mathrm{Fe}_{3} \mathrm{O}_{4}$ superparamagnetic cores were prepared by the chemical coprecipitation method. The $\mathrm{Fe}_{3} \mathrm{O}_{4}$ cores were synthesized with the mixed solution of ferric chloride hexahydrate $\left(\mathrm{FeCl}_{3} \cdot 6 \mathrm{H}_{2} \mathrm{O}\right)$ and ferric chloride tetrahydrate $\left(\mathrm{FeCl}_{2} \cdot 4 \mathrm{H}_{2} \mathrm{O}\right)$ at $80^{\circ} \mathrm{C}$ for $1 \mathrm{~h}$, together with a suitable amount of ammonia $\left(\mathrm{NH}_{4} \mathrm{OH}\right)$. The solution was centrifuged and washed with distilled water, and the $\mathrm{Fe}_{3} \mathrm{O}_{4}$ cores were dried for $12 \mathrm{~h}$. The average size of the prepared $\mathrm{Fe}_{3} \mathrm{O}_{4}$ cores is around 8-10 nm.

2.2. Synthesis of $\mathrm{SiO}_{2} / \mathrm{Fe}_{3} \mathrm{O}_{4}$ Nanoparticle. The $\mathrm{SiO}_{2}$ intermediary layer was prepared by using the sol-gel method. The above $\mathrm{Fe}_{3} \mathrm{O}_{4}$ cores were mixed with ethanol $(50 \mathrm{~mL})$ and ammonia under vigorous magnetic stirring. TEOS was added into the solution slowly and then aged for $5 \mathrm{~h}$. After

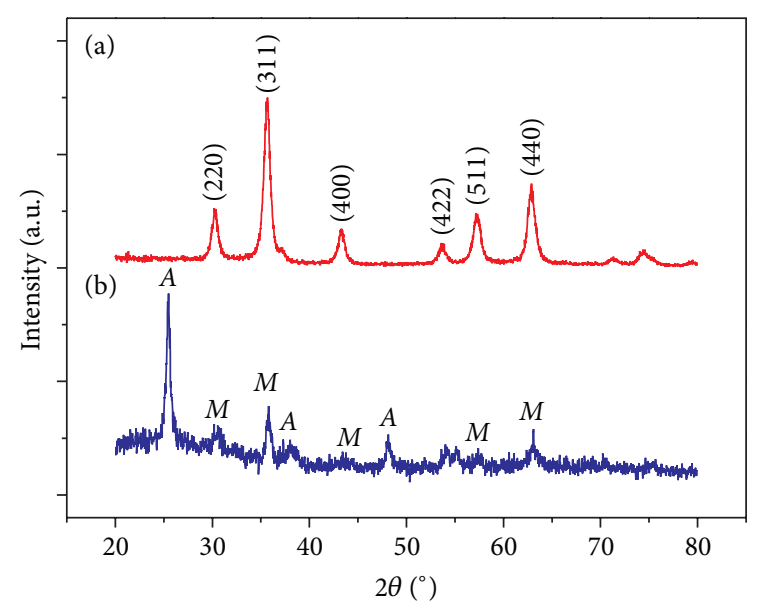

FIGURE 1: (a) XRD patterns of $\mathrm{Fe}_{3} \mathrm{O}_{4}$ core and (b) TSF photocatalyst. $A$ : anatase and $M$ : magnetite.

being washed with ethanol three times, $\mathrm{SiO}_{2} / \mathrm{Fe}_{3} \mathrm{O}_{4}$ (SF) nanoparticle was dispersed in ethanol for using.

2.3. Synthesis of $\mathrm{TiO}_{2} / \mathrm{SiO}_{2} / \mathrm{Fe}_{3} \mathrm{O}_{4}$ Photocatalyst. The outer layer $\mathrm{TiO}_{2}$ was prepared by using the sol-gel method. The $\mathrm{SiO}_{2} / \mathrm{Fe}_{3} \mathrm{O}_{4}$ (SF) nanoparticles were mixed with ethanol $(50 \mathrm{~mL})$ and ammonia under ultrasonic dispersion for $30 \mathrm{~min}$. An appropriate amount of TBOT was added into the solution drop by drop. The final photocatalysts were washed with ethanol three times, dried at $60^{\circ} \mathrm{C}$ for $48 \mathrm{~h}$, and finally were calcined at $450^{\circ} \mathrm{C}$ for $3 \mathrm{~h}$.

2.4. Characterization. The crystalline structure of particles was examined by using a Rigaku $D /$ max-RB X-ray diffraction (XRD) spectrometry with $\mathrm{Cu}-\mathrm{Ka}$ radiation. The size and microstructure of samples have been characterized with JEM 200CX transmission electron microscope (TEM) with $200 \mathrm{kV}$ operating voltage and JEOL-2011 high resolution transmission electron microscopy (HRTEM). The magnetic property of samples was measured with Lakeshore 7307 vibration sample magnetometer (VSM). The porosity of samples was measured by the nitrogen adsorption-desorption isotherm and $\mathrm{BJH}$ methods on the micromeritics ASAP 2000 specific surface area instrument. A UV-2802PC ultravioletvisible spectrometer was used to measure the UV/Vis absorption spectrum of the solutions to monitor the concentration of crude oil at different time intervals.

2.5. Photocatalytic Degradation of Crude Oily Wastewater. Photocatalytic activities of the nanoparticles have been evaluated by degradation experiments of modulated crude oily wastewater in a self-made photocatalytic reactor. An $8 \mathrm{~W}$ UV lamp was used as the ultraviolet light source and airblowing apparatus has been used; $0.2 \mathrm{~g} / \mathrm{L}$ TSF and $0.067 \mathrm{~g} / \mathrm{L}$ commercial $\mathrm{TiO}_{2}$ were suspended in the modulated crude 


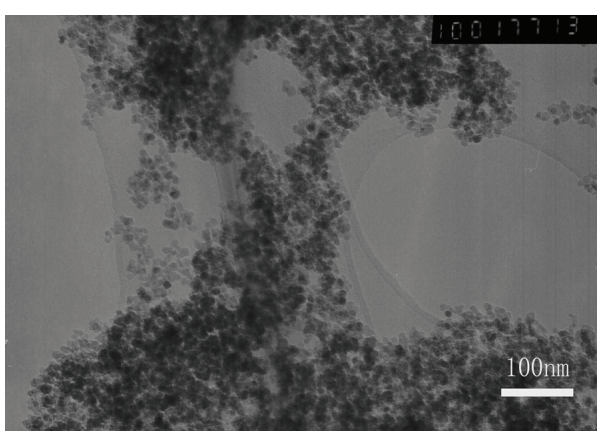

(a)

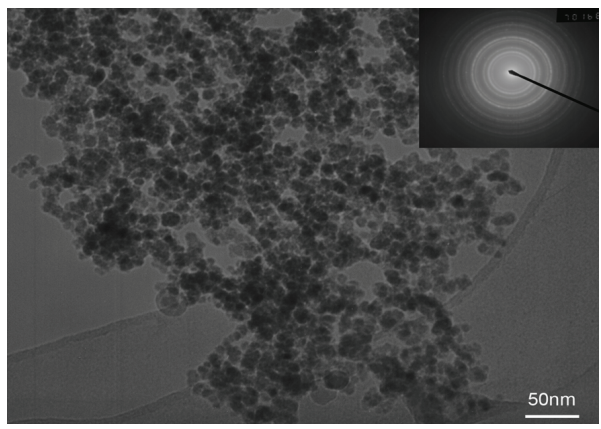

(c)

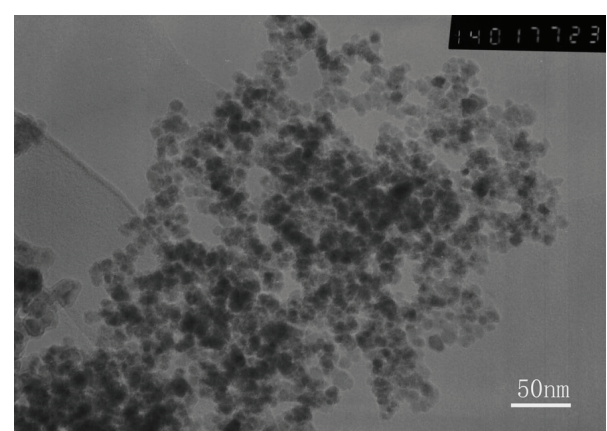

(b)

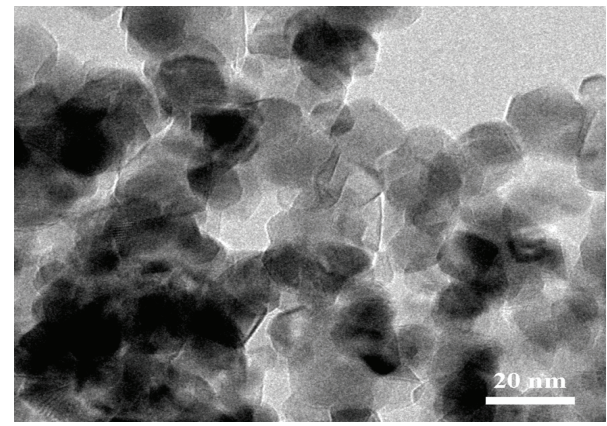

(d)

FIgURE 2: (a) TEM images of $\mathrm{Fe}_{3} \mathrm{O}_{4}$. (b) TEM images of SF. (c) TEM images of TSF. (d) HRTEM images of TSF. The inset in (c) is the electronic diffraction of TSF.

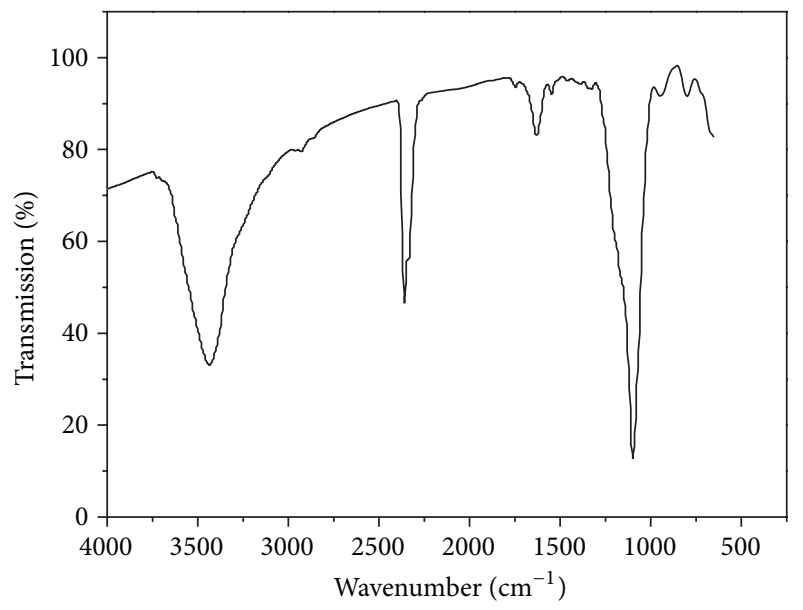

FIGURE 3: FTIR spectrum of TSF core-shell nanoparticles.

oily wastewater. To determine the change of crude oil concentration during photocatalysis process, a few milliliters of solution was taken from the mixture at different time. Then, the nanoparticles were separated from the solution with a magnetic bar. The solution was subsequently mixed with $\mathrm{CHCl}_{3}$, followed by centrifugation. Finally, $\mathrm{CHCl}_{3}$ solution was taken out and the oil concentration was measured with UV-Vis spectrometer (UV-2802PC Unico). According to the measurement, the oil concentration was calculated based on the concentration-absorbance curve obtained by a standard measurement.

\section{Results and Discussion}

3.1. Characterization of the Nanoparticles. The successful preparation of $\mathrm{Fe}_{3} \mathrm{O}_{4}$ magnetic nanoparticles and the TSF core-shell magnetic photocatalysts have been confirmed by $\mathrm{X}$-ray diffraction analysis (Figure 1 ). Figure $1(\mathrm{a})$ shows $\mathrm{X}$ ray diffraction patterns of $\mathrm{Fe}_{3} \mathrm{O}_{4}$ core. The diffraction peaks located at $30.0^{\circ}, 35.4^{\circ}, 43.0^{\circ}, 53.6^{\circ}, 57.2^{\circ}$, and $62.5^{\circ}$ can be perfectly indexed to the crystal planes (220), (311), (400), (422), (511), and (440) of magnetite phase $\mathrm{Fe}_{3} \mathrm{O}_{4}$, respectively. The average crystal size of $\mathrm{Fe}_{3} \mathrm{O}_{4}$ was calculated by using Scherrer's formula $D_{\mathrm{hkl}}=0.89 \lambda /(\beta \cos \theta)$, where $\beta$ is the width of the XRD peak at the half-peak height, $\lambda$ is the X-ray wavelength in nanometers, and $\theta$ is the half diffraction angle of $2 \theta$ in degrees. The average crystal size of $\mathrm{Fe}_{3} \mathrm{O}_{4}$, determined by the data from $D_{311}$, is around $11.7 \mathrm{~nm}$, which is approximately consistent with the TEM observation mentioned later. After two-step sol-gel processes and calcination, the X-ray spectra confirm the transformation of amorphous $\mathrm{TiO}_{2}$ to anatase, as shown in Figure 1(b). The diffraction peaks indicated by $A$ in Figure 1(b) are indexed as anatase $\mathrm{TiO}_{2}$, while diffraction peaks indicated by $M$ in Figure 1(b) are indexed as the magnetite $\mathrm{Fe}_{3} \mathrm{O}_{4}$. The wave packet that appears in the range of $20^{\circ} \sim 30^{\circ}$ might result from the interlayer of amorphous $\mathrm{SiO}_{2}$. No clear glassy sharp peak has been observed due to the fact that the thickness of the coated $\mathrm{SiO}_{2}$ layer is very small and the diffraction peak of amorphous structures is very weak if compared with the crystalline diffraction peaks. It approves that the coating layer of $\mathrm{Fe}_{3} \mathrm{O}_{4}$ is amorphous $\mathrm{SiO}_{2}$. " $A$ " peak is the diffraction peak of $\mathrm{TiO}_{2}\left(25.38^{\circ}, 48.04^{\circ}\right.$, and 


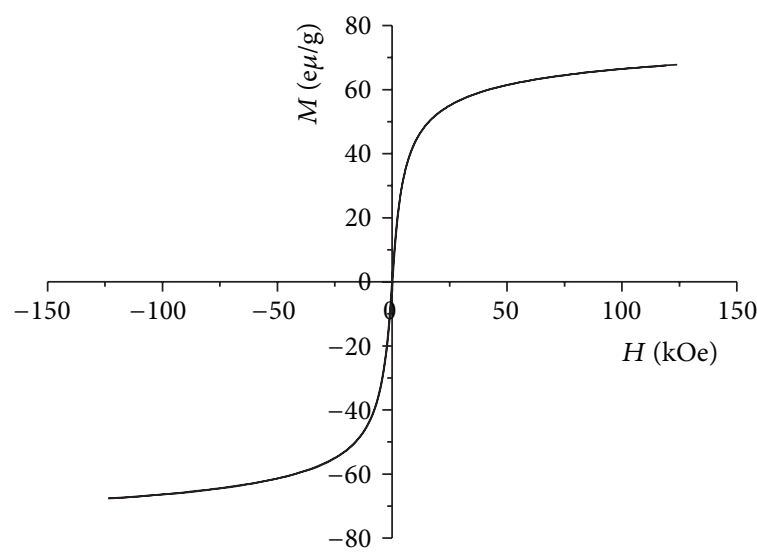

(a)

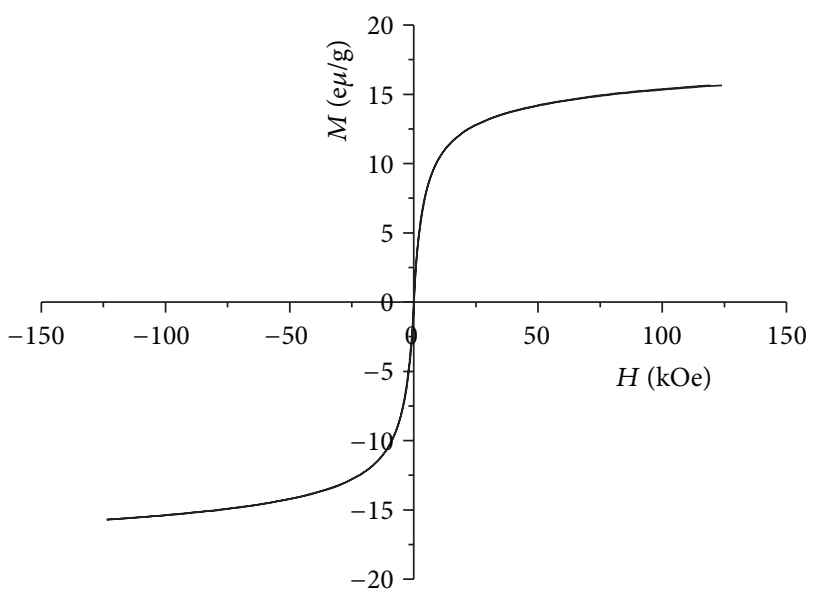

(b)

FIgURE 4: The magnetization curve of (a) $\mathrm{Fe}_{3} \mathrm{O}_{4}$ core and (b) TSF photocatalyst.

$37.80^{\circ}$ ), and it is the coincidence of three diffraction peaks of anatase, so it approves that the coating of SF nanoparticles is anatase $\mathrm{TiO}_{2}$. According to Scherrer's formula, the average TSF photocatalyst size was around $19.1 \mathrm{~nm}$.

The structure characterization of the as-prepared nanoparticles has been examined with transmission electron microscope (TEM) and high resolution transmission electron microscope (HRTEM). Figure 2(a) shows the morphology of $\mathrm{Fe}_{3} \mathrm{O}_{4}$ nanoparticles, and the size of $\mathrm{Fe}_{3} \mathrm{O}_{4}$ nanoparticles is around $5-20 \mathrm{~nm}$, which agreed with the result obtained from XRD analysis. The direct coating of silicon oxide onto the surface of the magnetite core was carried out by the hydrolysis of TEOS using sol-gel method, which can be seen from Figure 2(b). The SF nanoparticles had a core-shell structure in which magnetite was predominantly concentrated in the center of the SF nanoparticles. The direct coating of titanium oxide onto the surface of the SF nanoparticles was carried out by the hydrolysis of TBOT using sol-gel method, as can be seen from Figures 2(c) and 2(d). Before calcination, titanium oxide was amorphous and after calcination at $450^{\circ} \mathrm{C}$ for $3 \mathrm{~h}$, the amorphous $\mathrm{TiO}_{2}$ transformed to the anatase phase, which is confirmed by the peaks in the XRD pattern (Figure 1(b)). By using Scherrer's formula, the size of TSF photocatalyst is around $10-30 \mathrm{~nm}$, which matches the results of XRD analysis.

The Fourier transform infrared (FTIR) spectroscopy spectrum of the calcined sample was investigated to confirm the structure of core-shell nanoparticles. As shown in Figure 3, TSF core-shell nanoparticles show more signals than P25. The band at ca. $1620 \mathrm{~cm}^{-1}$ can be assigned to the $\mathrm{H}-\mathrm{O}-\mathrm{H}$ stretching vibration, and the band at ca. $1100 \mathrm{~cm}^{-1}$ and $800 \mathrm{~cm}^{-1}$ corresponds to the asymmetric vibration and symmetric vibration of $\mathrm{Si}-\mathrm{O}-\mathrm{Si}$. The presence of water is proved by the stretching mode at $3400 \mathrm{~cm}^{-1}$. This surface hydroxylation will benefit the photocatalytic degradation of organic contaminants. Notably, no absorption peaks corresponding to $\mathrm{Fe}_{3} \mathrm{O}_{4}$ core are revealed, suggesting that it was totally coated by outer shell.
The magnetic properties of the $\mathrm{Fe}_{3} \mathrm{O}_{4}$ nanoparticles and TSF core-shell magnetic nanoparticles have been measured by use of the vibration sample magnetometer. The magnetization curves of $\mathrm{Fe}_{3} \mathrm{O}_{4}$ nanoparticles and TSF coreshell nanoparticles are shown in Figures 4(a) and 4(b), respectively. According to Figure 4, the saturation magnetization and the residual magnetization of $\mathrm{Fe}_{3} \mathrm{O}_{4}$ nanoparticles are $67.7 \mathrm{emu} / \mathrm{g}$ and $1.6 \mathrm{emu} / \mathrm{g}$, respectively, while the coercivity of $\mathrm{Fe}_{3} \mathrm{O}_{4}$ core is close to zero, indicating the existence of superparamagnetism characteristics. After twostep coating and calcination, the saturation magnetization and the residual magnetization of TSF photocatalyst are $16.7 \mathrm{emu} / \mathrm{g}$ and $0.74 \mathrm{emu} / \mathrm{g}$, respectively, while the coercivity of TSF photocatalyst is $5.78 \mathrm{Gs}$, which is still close to zero, confirming the superparamagnetism nature of TSF photocatalyst. Due to the superparamagnetism nature, TSF photocatalyst can demagnetize easily. When additional magnetic field intensity decreases to zero, the residual magnetization of TSF photocatalyst also drops to zero quickly, which would benefit the removal and demagnetization of TSF photocatalyst. It would provide the feasibility of the recovery and reusing of TSF photocatalyst.

The surface area of TSF photocatalyst and P25 was investigated by using nitrogen adsorption-desorption isotherms. The calculated BET surface area of TSF photocatalyst and P25 is $40.5 \mathrm{~m}^{2} / \mathrm{g}$ and $50 \mathrm{~m}^{2} / \mathrm{g}$, respectively. It is known that large specific surface area is beneficial to the photocatalytic activity.

3.2. Photocatalytic Activity. We further demonstrated the photocatalytic performance of TSF photocatalyst by the removal of crude oil from oily wastewater. For comparison, we also evaluated the photocatalytic performance of P25 anatase $\mathrm{TiO}_{2}$. Figure 5(a) shows the absorption spectra of oily wastewater exposed to UV light for different time intervals. The typical absorption peak at $245 \mathrm{~nm}$ gradually decreases as the time increases and completely disappears after $40 \mathrm{~min}$, implying the complete degradation of crude 


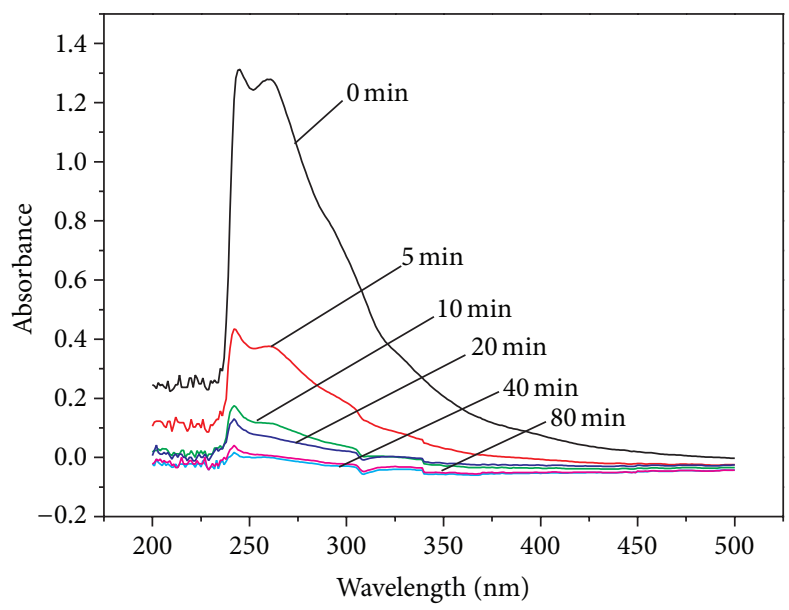

(a)

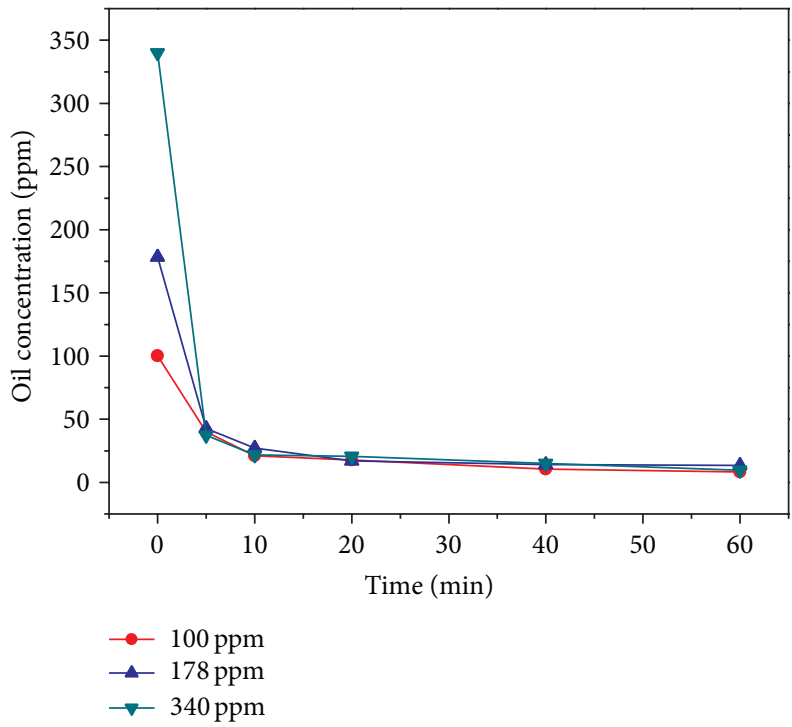

(c)

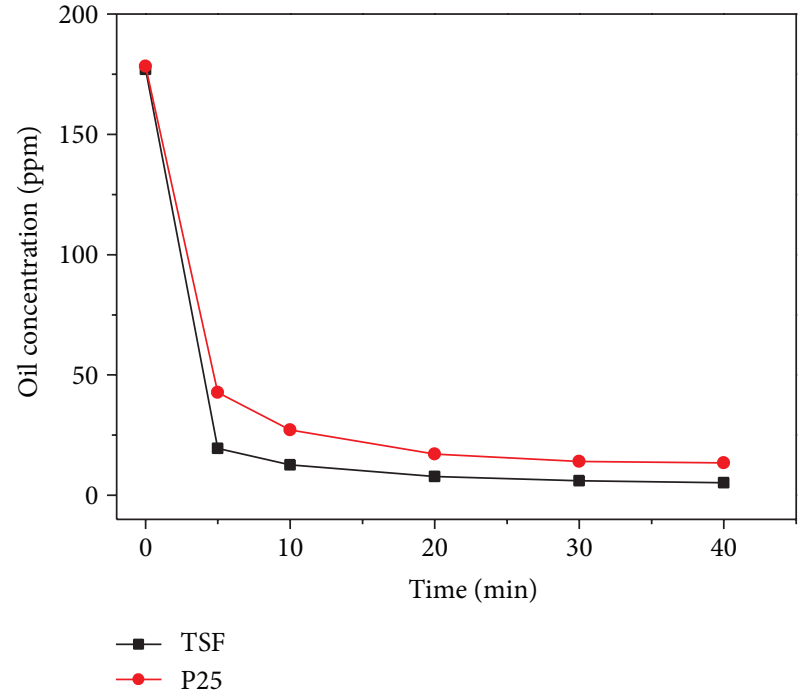

(b)

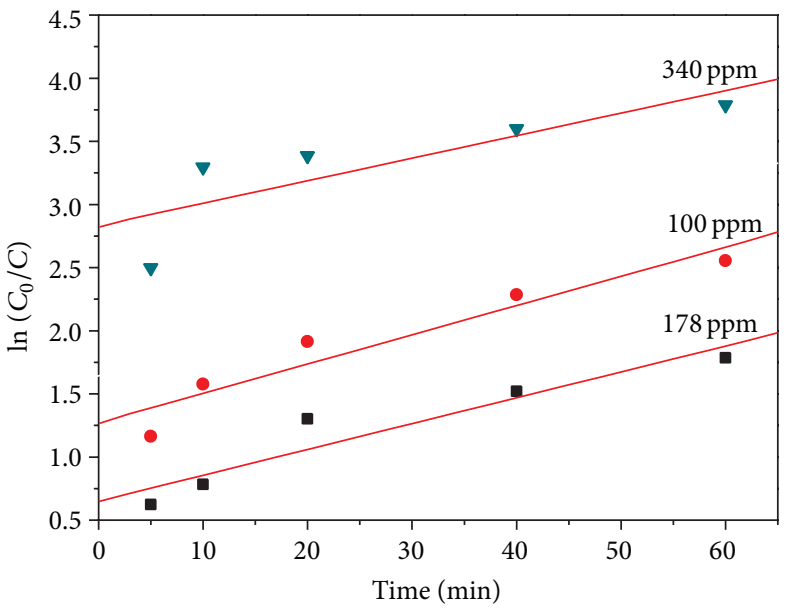

$178 \mathrm{ppm}$

$100 \mathrm{ppm}$

จ $340 \mathrm{ppm}$

Figure 5: (a) Absorption spectra of oily wastewater exposed to UV light. (b) Photocatalytic performance of TSF and P25. (c) The effect of initial oil concentration on the photocatalysis process of TSF. (d) Pseudo-first-order model fitting of (c) in degrading crude oily wastewater with the oil concentration less than $350 \mathrm{ppm}$.

oil by the photocatalysts. Figure 5(b) shows that the oil concentration of oily wastewater varies with the photocatalytic degrading time. It shows that with the increase of degrading time the oil concentration decreases rapidly. After 10 min photocatalytic degradation, the oil concentration is reduced from 176 to $20 \mathrm{ppm}$. It indicates that the TSF coreshell magnetic nanoparticles possess high photocatalytic activity and can purify the crude oily wastewater efficiently. While keeping the amount of $\mathrm{TiO}_{2}$ in TSF photocatalyst and P25 $\mathrm{TiO}_{2}$ the same, we found that TSF photocatalyst and P25 $\mathrm{TiO}_{2}$ show similar photocatalytic activity under identical conditions, as shown in Figure 5(b). The good photocatalytic activity of TSF photocatalyst may be caused by two reasons.
One reason is the small size of anatase phase formed during the calcination process. Some previous papers pointed out the optimal size of anatase for photocatalysis is around $10 \mathrm{~nm}$ [27-29]. The other reason is the formation of Ti-O-Si bond, which has been reported in many papers to enhance the photocatalytic efficiency [30-32]. Meanwhile, $\mathrm{SiO}_{2}$ intermediary layer inhibits electrical contact and prevents photogenerated electrons from transferring into the lower lying conduction band of the iron oxide core, thus eliminating the possible photodissolution of iron oxide in the reaction process.

Figure 5(c) shows that the curves of oil concentration vary with photocatalytic degradation time for different initial oil concentration in the wastewater. It has been found that 
TABLE 1: Kinetic parameter of oil degradation.

\begin{tabular}{llcc}
\hline $\begin{array}{l}\text { Initial oil concentration } \\
C_{0}(\mathrm{ppm})\end{array}$ & $\begin{array}{c}\text { Pseudo-first-order kinetics } \\
\text { equation }\end{array}$ & $\begin{array}{c}\text { Kinetic constant } k_{\mathrm{obs}} \\
\left(\mathrm{min}^{-1}\right)\end{array}$ & $\begin{array}{c}\text { Correlation coefficient } R^{2} \\
100\end{array}$ \\
178 & $\ln \left(C_{0} / C\right)=1.2730+0.0231 t$ & 0.0231 & 0.9554 \\
340 & $\ln \left(C_{0} / C\right)=0.6504+0.0204 t$ & 0.0204 & 0.9566 \\
& $\ln \left(C_{0} / C\right)=2.8314+0.0178 t$ & 0.0178 & 0.8227 \\
\hline
\end{tabular}

even the initial oil concentration is as high as $340 \mathrm{ppm}$, the crude oily wastewater could be purified quickly and the oil concentration can be reduced to less than $30 \mathrm{ppm}$ within 20 minutes. It indicates that TSF nanoparticles exhibit high efficiency. It has been shown that the photocatalytic degradation of crude oil follows pseudo-first-order kinetics according to Langmuir-Hinshelwood model, and the photocatalytic reaction can be described simply by $\ln \left(C_{0} / C\right)=k t$, where $C$ and $C_{0}$ are the actual and initial oil concentration and $k$ is the degradation rate parameter. The kinetic parameter and pseudo-first-order model fitting are summarized in Table 1 and Figure 5(d). The sample with initial oil concentration 100 ppm exhibits the highest photocatalytic efficiency; its kinetic constant $K_{\mathrm{obs}}$ is $0.0231 \mathrm{~min}^{-1}$, which is much higher than that with initial oil concentration of $178 \mathrm{ppm}$ or $340 \mathrm{ppm}$. The increase of oil concentration leads to a decrease in photocatalytic activity.

3.3. Recovery Properties. After photocatalytic degradation experiments, the TSF core-shell magnetic nanoparticles within the wastewater in the container could be easily withdrawn with an external magnetic field ( $\mathrm{NdFeB}$ magnet). When the magnet is located at the bottom outside of the container, all of the TSF core-shell magnetic nanoparticles could be separated from the wastewater and attracted to the bottom of the container within only 5 minutes. The photocatalytic activity of the recovered photocatalysts has not noticeably changed after three successive cycles under UV-Vis irradiation indicating that magnetically recoverable photocatalyst is stable and effective for the degradation of crude oil. The photocatalytic performance of TSF after three cycles under UV-Vis irradiation is illustrated in Figure 6. The slight decrease in photocatalytic activity might result from the absorbance of crude oil, but it could be recovered by a high temperature calcination of the contaminant. Present results indicate that the magnetically recoverable TSF coreshell nanoparticles are stable and effective for the degradation of crude oily wastewater.

\section{Conclusions}

In summary, TSF core-shell magnetic nanoparticles, which are constituted by a $\mathrm{Fe}_{3} \mathrm{O}_{4}$ core, a $\mathrm{SiO}_{2}$ protective intermediary layer, and a $\mathrm{TiO}_{2}$ outshell, have been prepared by use of chemical coprecipitation and sol-gel processes. The as-prepared TSF core-shell magnetic nanoparticles display superparamagnetic behavior with saturated magnetization of $16.7 \mathrm{eum} / \mathrm{g}$, residue magnetization of $0.74 \mathrm{eum} / \mathrm{g}$, and coercivity of $5 \mathrm{Gs}$. It has been found that the TSF core-shell

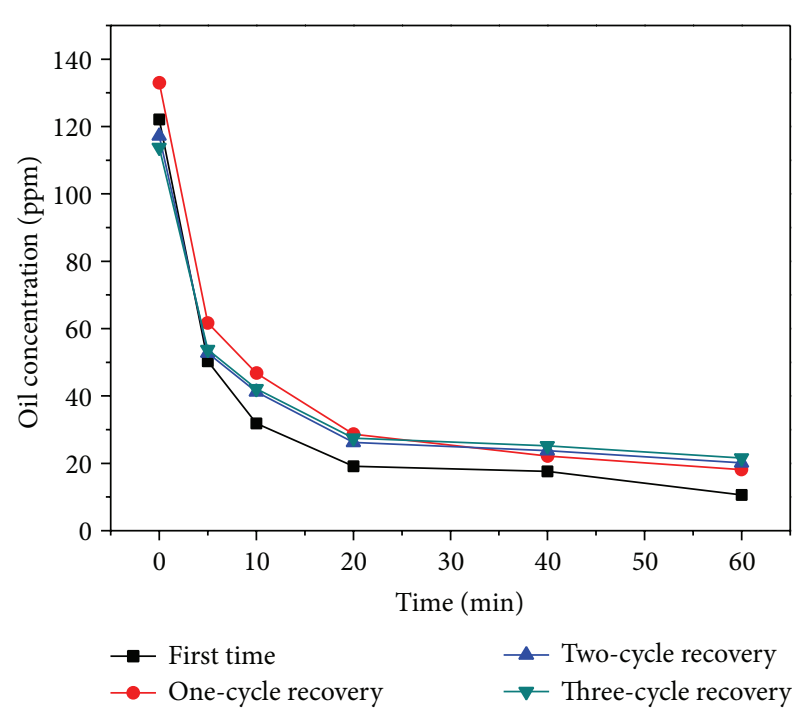

FIgURE 6: Photocatalytic performance of TSF after three cycles.

magnetic nanoparticles possess high photocatalytic activity in the degradation of crude oily wastewater. With the addition of $0.2 \mathrm{~g} / \mathrm{L}$ TSF core-shell magnetic nanoparticles, the oil concentration in the crude oily wastewater could be reduced to lower than $30 \mathrm{ppm}$ within 20 minutes for the initial oil concentration less than $350 \mathrm{ppm}$. After photocatalytic experiments, the TSF core-shell magnetic nanoparticles could be easily separated from the wastewater and withdrawn back by use of external magnetic field (a NdFeB magnet). It has been found that recovered TSF core-shell magnetic nanoparticles also exhibit high efficiency in the degradation of crude oily wastewater even for three cycles. Present results indicate that magnetically recoverable TSF core-shell magnetic nanoparticles are promising nanomaterials for degrading the crude oily wastewater.

\section{Conflict of Interests}

The authors declare that there is no conflict of interests regarding the publication of this paper.

\section{Acknowledgments}

This work is supported by the Jiangsu University Scientific Research Foundation for Advanced Talents (Grant no. 12JDG095) and the National Natural Science Foundation of China (Grant no. 51302112). 


\section{References}

[1] M. R. Hoffmann, S. T. Martin, W. Choi, and D. W. Bahnemann, "Environmental applications of semiconductor photocatalysis," Chemical Reviews, vol. 95, no. 1, pp. 69-96, 1995.

[2] O. Carp, C. L. Huisman, and A. Reller, "Photoinduced reactivity of titanium dioxide," Progress in Solid State Chemistry, vol. 32, no. 1-2, pp. 33-177, 2004.

[3] A. L. Linsebigler, G. Lu, and J. T. Yates Jr., "Photocatalysis on $\mathrm{TiO}_{2}$ surfaces: principles, mechanisms, and selected results," Chemical Reviews, vol. 95, no. 3, pp. 735-758, 1995.

[4] H. G. Yu, R. Liu, X. F. Wang, P. Wang, and J. Yu, "Enhanced visible-light photocatalytic activity of $\mathrm{Bi}_{2} \mathrm{WO}_{6}$ nanoparticles by $\mathrm{Ag}_{2} \mathrm{O}$ cocatalyst," Applied Catalysis B: Environmental, vol. 111112, pp. 326-333, 2012.

[5] P. Wang, J. Wang, X. F. Wang, H. G. Yu, J. G. Yu, and M. Lei, "One-step synthesis of easy-recycling $\mathrm{TiO}_{2}-\mathrm{rGO}$ nanocomposite photocatalysts with enhanced photocatalytic activity," Applied Catalysis B: Environmental, vol. 132-133, pp. 452-459, 2013.

[6] J. G. Yu, Y. Wang, and W. Xiao, "Enhanced photoelectrocatalytic performance of $\mathrm{SnO}_{2} / \mathrm{TiO}_{2}$ rutile composite films," Journal of Materials Chemistry A, vol. 1, no. 36, pp. 10727-10735, 2013.

[7] R. Wang, K. Hashimoto, A. Fujishima et al., "Light-induced amphiphilic surfaces," Nature, vol. 388, no. 6641, pp. 431-432, 1997.

[8] R. Liu, P. Wang, X. F. Wang, H. G. Yu, and J. G. Yu, "UV- and visible-light photocatalytic activity of simultaneously deposited and doped $\mathrm{Ag} / \mathrm{Ag}(\mathrm{I})-\mathrm{TiO}_{2}$ photocatalyst," Journal of Physical Chemistry C, vol. 116, no. 33, pp. 17721-17728, 2012.

[9] P. Zhou, J. Yu, and Y. X. Wang, "The new understanding on photocatalytic mechanism of visible-light response N-S codoped anatase $\mathrm{TiO}_{2}$ by first- principles," Applied Catalysis B: Environmental, vol. 142, pp. 45-53, 2013.

[10] S. C. Roy, O. K. Varghese, M. Paulose, and C. A. Grimes, "Toward solar fuels: photocatalytic conversion of carbon dioxide to hydrocarbons," ACS Nano, vol. 4, no. 3, pp. 1259-1278, 2010.

[11] X. Chen and S. S. Mao, "Titanium dioxide nanomaterials: synthesis, properties, modifications and applications," Chemical Reviews, vol. 107, no. 7, pp. 2891-2959, 2007.

[12] D. Chen and J. Ye, "Hierarchical $\mathrm{WO}_{3}$ hollow shells: dendrite, sphere, dumbbell, and their photocatalytic properties," Advanced Functional Materials, vol. 18, no. 13, pp. 1922-1928, 2008.

[13] S. Watson, J. Scott, D. Beydoun, and R. Amal, "Studies on the preparation of magnetic photocatalysts," Journal of Nanoparticle Research, vol. 7, no. 6, pp. 691-705, 2005.

[14] J. M. Lee, M. S. Kim, and B. W. Kim, "Photodegradation of bisphenol-A with $\mathrm{TiO}_{2}$ immobilized on the glass tubes including the UV light lamps," Water Research, vol. 38, no. 16, pp. 3605-3613, 2004.

[15] D. Beydoun, R. Amal, G. K.-C. Low, and S. McEvoy, "Novel photocatalyst: titania-coated magnetite. Activity and photodissolution," Journal of Physical Chemistry B, vol. 104, no. 18, pp. 4387-4396, 2000.

[16] R. Guo, L. Fang, W. Dong, F. Zheng, and M. Shen, "Magnetically separable $\mathrm{BiFeO}_{3}$ nanoparticles with a $\gamma-\mathrm{Fe}_{2} \mathrm{O}_{3}$ parasitic phase: controlled fabrication and enhanced visible-light photocatalytic activity," Journal of Materials Chemistry, vol. 21, no. 46, pp. 18645-18652, 2011.
[17] S. Watson, D. Beydoun, and R. Amal, "Synthesis of a novel magnetic photocatalyst by direct deposition of nanosized $\mathrm{TiO}_{2}$ crystals onto a magnetic core," Journal of Photochemistry and Photobiology A: Chemistry, vol. 148, no. 1-3, pp. 303-313, 2002.

[18] N. Quici, M. E. Morgada, R. T. Gettar, M. Bolte, and M. I. Litter, "Photocatalytic degradation of citric acid under different conditions: $\mathrm{TiO}_{2}$ heterogeneous photocatalysis against homogeneous photolytic processes promoted by $\mathrm{Fe}(\mathrm{III})$ and $\mathrm{H}_{2} \mathrm{O}_{2}$," Applied Catalysis B: Environmental, vol. 71, no. 3-4, pp. 117-124, 2007.

[19] S.-K. Li, F.-Z. Huang, Y. Wang et al., "Magnetic $\mathrm{Fe}_{3} \mathrm{O}_{4} @ \mathrm{C} @ \mathrm{Cu} 2 \mathrm{O}$ composites with bean-like core/shell nanostructures: synthesis, properties and application in recyclable photocatalytic degradation of dye pollutants," Journal of Materials Chemistry, vol. 21, no. 20, pp. 7459-7466, 2011.

[20] S. Xu, W. Shangguan, J. Yuan, M. Chen, J. Shi, and Z. Jiang, "Synthesis and performance of novel magnetically separable nanospheres of titanium dioxide photocatalyst with egg-like structure," Nanotechnology, vol. 19, no. 9, Article ID 095606, 2008.

[21] Y. S. Chung, S. B. Park, and D. Kang, "Magnetically separable titania-coated nickel ferrite photocatalyst," Materials Chemistry and Physics, vol. 86, no. 2-3, pp. 375-381, 2004.

[22] D. Beydoun, R. Amal, G. Low, and S. McEvoy, "Occurrence and prevention of photodissolution at the phase junction of magnetite and titanium dioxide," Journal of Molecular Catalysis A: Chemical, vol. 180, no. 1-2, pp. 193-200, 2002.

[23] D. Beydoun and R. Amal, "Implications of heat treatment on the properties of a magnetic iron oxide-titanium dioxide photocatalyst," Materials Science and Engineering B: Solid-State Materials for Advanced Technology, vol. 94, no. 1, pp. 71-81, 2002.

[24] Y. Gao, B. Chen, H. Li, and Y. Ma, "Preparation and characterization of a magnetically separated photocatalyst and its catalytic properties," Materials Chemistry and Physics, vol. 80, no. 1, pp. 348-355, 2003.

[25] D. Beydoun, R. Amal, J. Scott, G. Low, and S. McEvoy, "Studies on the mineralization and separation efficiencies of a magnetic photocatalyst," Chemical Engineering \& Technology, vol. 24, no. 7, pp. 745-748, 2001.

[26] F. Chen, Y. Xie, J. Zhao, and G. Lu, "Photocatalytic degradation of dyes on a magnetically separated photocatalyst under visible and UV irradiation," Chemosphere, vol. 44, no. 5, pp. 1159-1168, 2001.

[27] S. Y. Chae, M. K. Park, S. K. Lee, T. Y. Kim, S. K. Kim, and W. I. Lee, "Preparation of size-controlled $\mathrm{TiO}_{2}$ nanoparticles and derivation of optically transparent photocatalytic films," Chemistry of Materials, vol. 15, no. 17, pp. 3326-3331, 2003.

[28] J. Grzechulska and A. W. Morawski, "Photocatalytic labyrinth flow reactor with immobilized $\mathrm{P} 25 \mathrm{TiO}_{2}$ bed for removal of phenol from water," Applied Catalysis B: Environmental, vol. 46, no. 2, pp. 415-419, 2003.

[29] S. Anandan and M. Yoon, "Photocatalytic activities of the nanosized $\mathrm{TiO}_{2}$-supported Y-zeolites," Journal of Photochemistry and Photobiology C: Photochemistry Reviews, vol. 4, no. 1, pp. 5-18, 2003.

[30] K. Qi, X. Chen, Y. Liu, J. H. Xin, C. L. Mak, and W. A. Daoud, "Facile preparation of anatase $/ \mathrm{SiO}_{2}$ spherical nanocomposites and their application in self-cleaning textiles," Journal of Materials Chemistry, vol. 17, no. 33, pp. 3504-3508, 2007.

[31] L. Zhou, S. Yan, B. Tian, J. Zhang, and M. Anpo, "Preparation of $\mathrm{TiO}_{2}-\mathrm{SiO}_{2}$ film with high photocatalytic activity on PET substrate," Materials Letters, vol. 60, no. 3, pp. 396-399, 2006. 
[32] A. Matsuda, T. Matoda, Y. Kotani, T. Kogure, M. Tatsumisago, and T. Minami, "Evaluation of photocatalytic activity of transparent anatase nanocrystals-dispersed silica films prepared by the sol-gel process with hot water treatment," Journal of Sol-Gel Science and Technology, vol. 26, no. 1-3, pp. 517-521, 2003. 

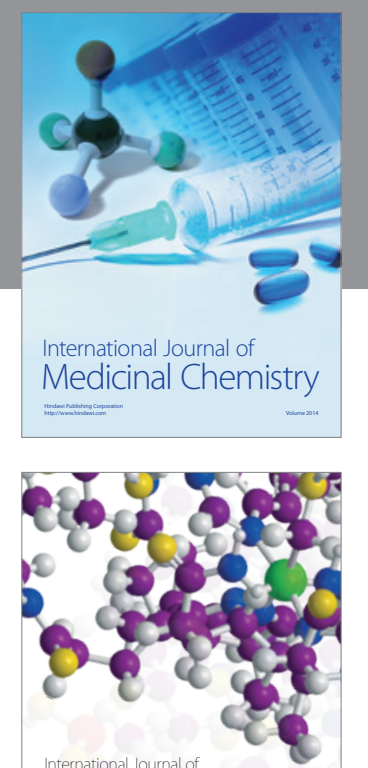

\section{Carbohydrate} Chemistry

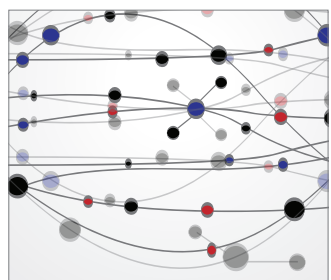

The Scientific World Journal
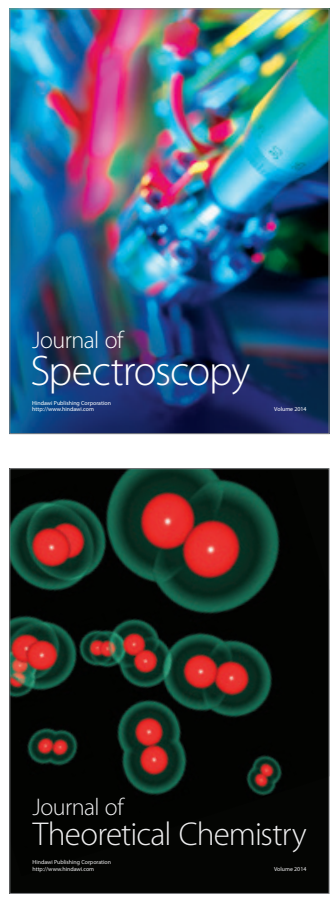
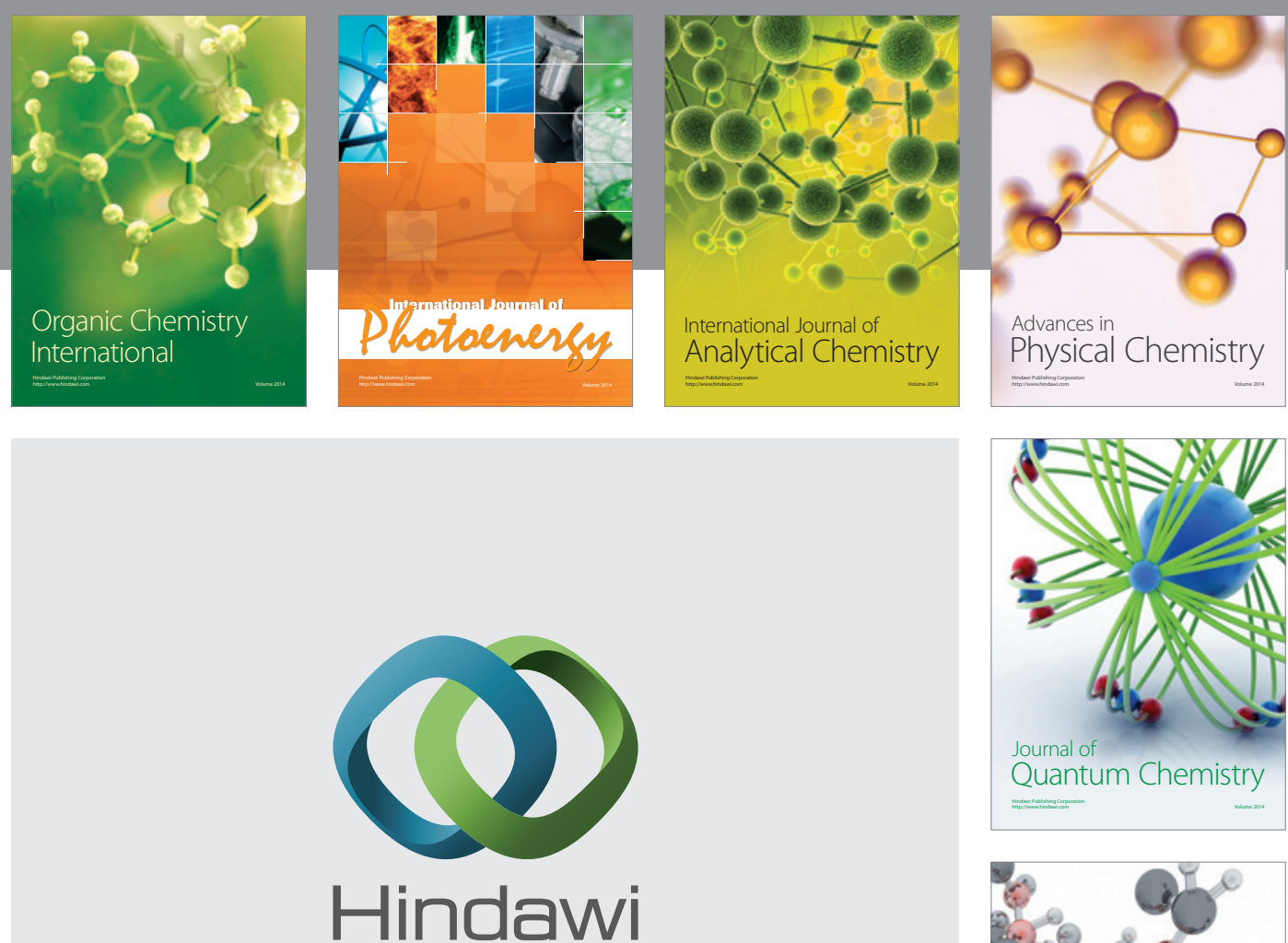

Submit your manuscripts at

http://www.hindawi.com

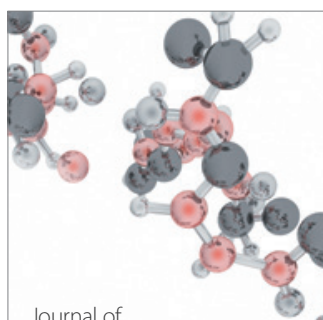

Analytical Methods

in Chemistry

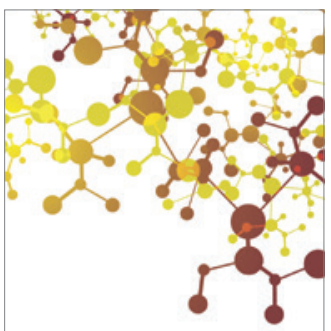

Journal of

Applied Chemistry

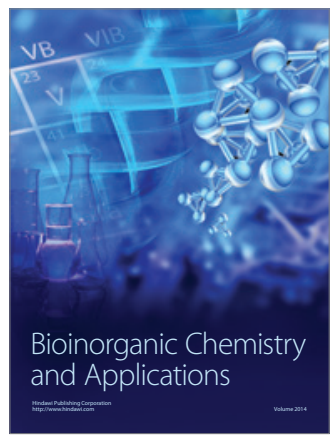

Inorganic Chemistry
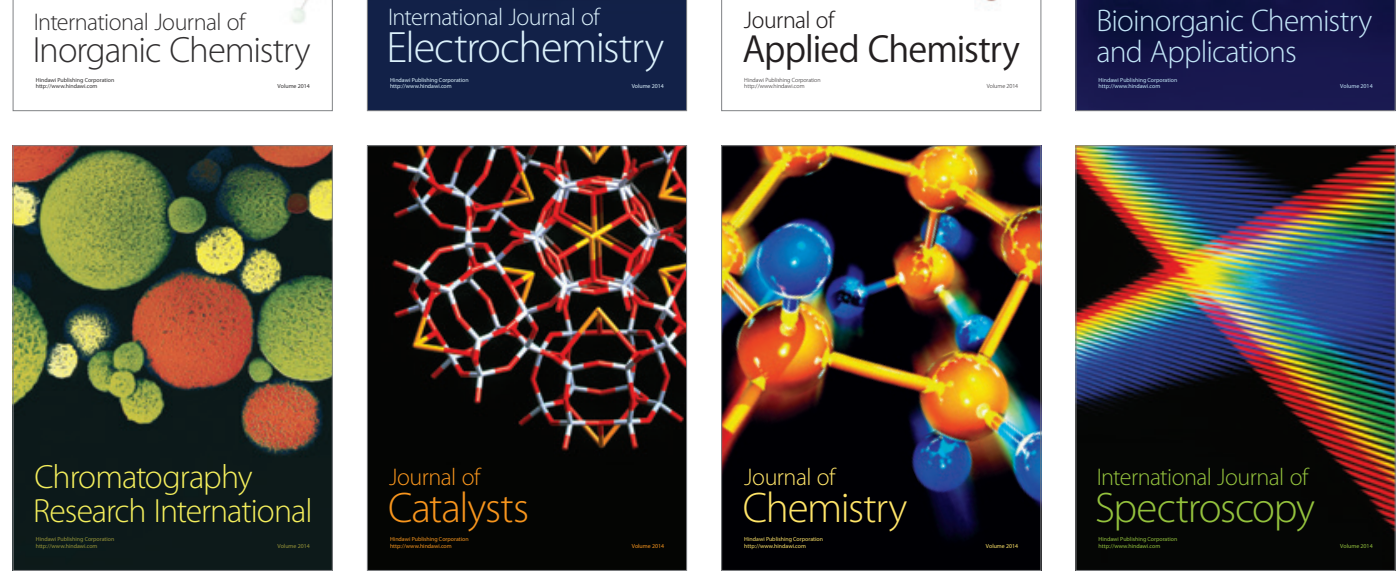